\title{
When to Perform UDT After SCI
}

There is no fixed time after SCI when the first UDT should be done. It depends on many factors: when patients are admitted, complications as UTI that need to be cured first, availability of UDT personnel, room and equipment. One should aim to get information after the period of spinal shock of the LUT, but it is not seldom uncertain when this period has ended.

Mostly the first UDT is done on average at 2 months after SCI. With very early transfer to the rehab unit and when neurological examination shows active sacral reflexes for suprasacral lesions or absence of such reflexes in cases of sacral/conal lesions, the first test, done at 4-6 weeks, can already provide important data. If, for one reason or another, the first UDT can only be performed at 4 or at 6 months post SCI, its clinical value is still high. 